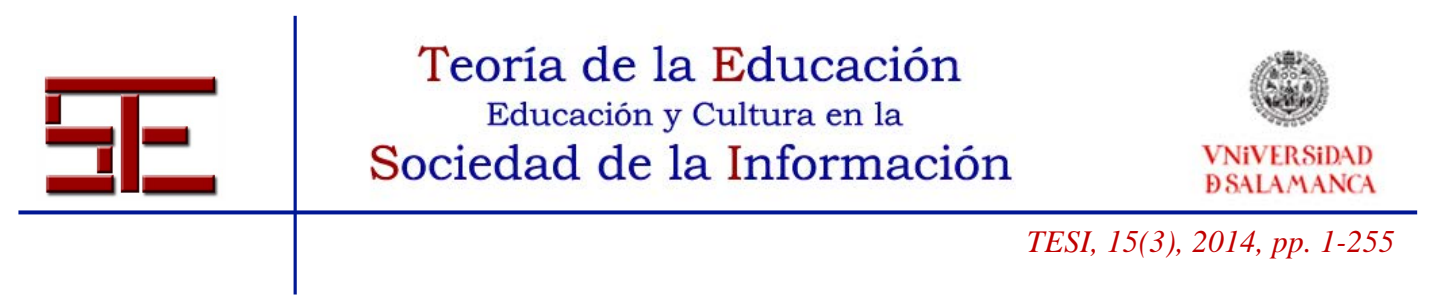

\title{
DESARROLLO DE HABILIDADES SOCIALES EN ESTUDIANTES MEXICANOS DE PREPARATORIA A TRAVÉS DE ACTIVIDADES VIRTUALES EN LA PLATAFORMA MOODLE
}

\author{
Laura Yolanda \\ RODRÍGUEZ \\ MATAMOROS \\ Doctoranda en Educación \\ Procuradora de Derechos \\ Universitarios \\ Rectoría, \\ Universidad Iberoamericana \\ Puebla \\ México \\ DXUURGU XH] \# IEHRSXHEDDP [ [
}

\section{María Luz CACHEIRO GONZÁLEZ}

Doctora en Educación

Profesora del Departamento de

Didáctica, Organización Escolar y

Didácticas Especiales

Facultad de Educación

Universidad Nacional de Educación a

Distancia $\square$ ( VSDD

P GLFKHIR\# HGXIXQHATH
Juan Antonio GIL PASCUAL

Doctor en Educación

Profesor del Departamento de

Métodos de Investigación y

Diagnóstico en Educación

Facultad de Educación

Universidad Nacional de

Educación a

Distancia. España

jgil@edu.uned.es

\section{Resumen:}

Con la intención de promover las habilidades sociales de estudiantes mexicanos de preparatoria con base en el perfil de egreso de este nivel educativo, se aplicaron actividades virtuales en la plataforma Moodle a 169 estudiantes de segundo grado, adoptando la propuesta de habilidades sociales de Goldstein. Con la intención de establecer el impacto de estas actividades se empleó un diseño pretest-postest de un solo grupo. Los resultados muestran que las actividades tuvieron un impacto positivo y significativo en las habilidades sociales básicas, las habilidades sociales avanzadas, las habilidades para el manejo de sentimientos y las habilidades sociales alternativas de los participantes de acuerdo a los resultados obtenidos mediante la escala de habilidades sociales de Goldstein.

Palabras clave: Habilidades sociales básicas, habilidades sociales avanzadas, habilidades para el manejo de sentimientos, habilidades sociales alternativas, plataforma Moodle. 


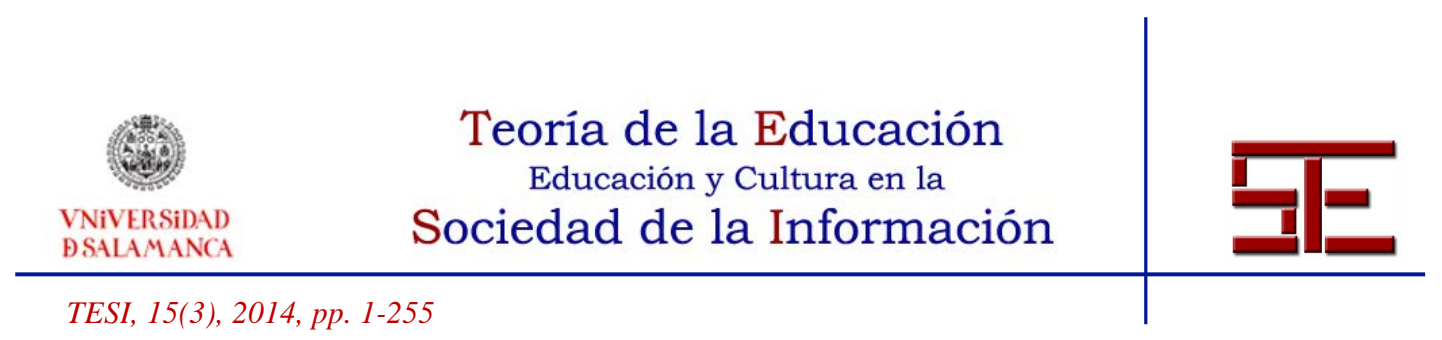

PROMOTING SOCIAL SKILLS OF MEXICAN HIGH SCHOOL STUDENTS THROUGH VIRTUAL ACTIVITIES IN THE MOODLE PLATFORM

\begin{abstract}
:
With the intention of promoting social skills of Mexican high school students based on the graduate profile of this level, virtual activities were implemented in the Moodle platform to 169 students of second year, adopting the proposed Goldstein social skills. In order to establish the impact of these activities to a pretest-postest a one group design was used. The results show that the activities had a positive and significant impact in beginning social skills, advanced social skills, skills for dealing with feeling, social alternatives skills of the participants according to the results obtained by skills scale social Goldstein.
\end{abstract}

Key words: Basic social skills, advanced social skills, skills for dealing with feeling, alternatives social skills, Moodle platform. 


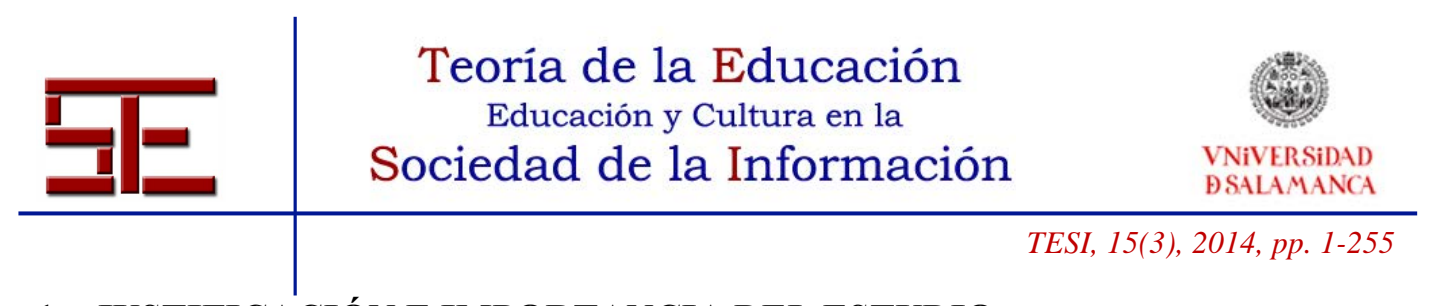

\section{JUSTIFICACIÓN E IMPORTANCIA DEL ESTUDIO}

El perfil de egreso de preparatoria en México considera el desarrollo de habilidades sociales relacionadas con el trabajo en equipo, la apertura al diálogo, la participación social responsable, la resolución no-violenta de conflictos, la conciencia cívica y ética, el respeto de los derechos de los demás, entre otros (SEP, 2008). El desarrollo de las habilidades sociales contempladas en este perfil resulta relevante si se considera que en la medida que los estudiantes cuenten con herramientas para entablar relaciones interpersonales sanas y nutricias podrán mejorar su proyección personal, profesional y social.

Sin embargo, al examinar los programas de las asignaturas que componen el plan de estudios de una preparatoria pública del Estado de Puebla, México, no se identificaron acciones didácticas encaminadas al desarrollo de las habilidades sociales consideradas en el perfil de egreso de este nivel educativo.

Adicionalmente, los directivos y profesores de esta preparatoria reportan comportamientos antisociales por parte de sus estudiantes consistentes en timidez y retraimiento, riñas entre compañeros, resistencia al trabajo en equipo y actitudes retadoras ante la autoridad, entre otros. También mencionan recibir reportes de los padres de estos estudiantes en los que indican que sus hijos muestran comportamientos rebeldes, aislamiento, poca participación en las tareas del hogar, falta de comunicación, entre otros.

De ahí que se considere importante promover el desarrollo de las habilidades sociales en los estudiantes de segundo grado de esta preparatoria pública.

La principal aportación del presente estudio consiste en el desarrollo de actividades virtuales en la plataforma Moodle orientadas a la promoción de habilidades sociales que respondan a los perfiles, características y requerimientos de estudiantes mexicanos de segundo grado de preparatoria con la intención de favorecer un comportamiento prosocial.

En este artículo se presenta en un primer momento el objeto de estudio en el que se plantean las preguntas de investigación, la hipótesis y la definición de las variables, así como el objetivo, el contexto, el público-objetivo, el tipo de estudio y el instrumento utilizado. En un segundo momento se exponen los principales referentes teóricos en los que se enmarca la investigación. Posteriormente, se describe el desarrollo del estudio presentando aspectos generales del diseño instruccional de las actividades virtuales. Después se presentan los resultados más relevantes para cerrar con las conclusiones del estudio destacando aspectos tales como la sostenibilidad de la propuesta, su transferibilidad a otros contextos y algunas recomendaciones para su aplicación.

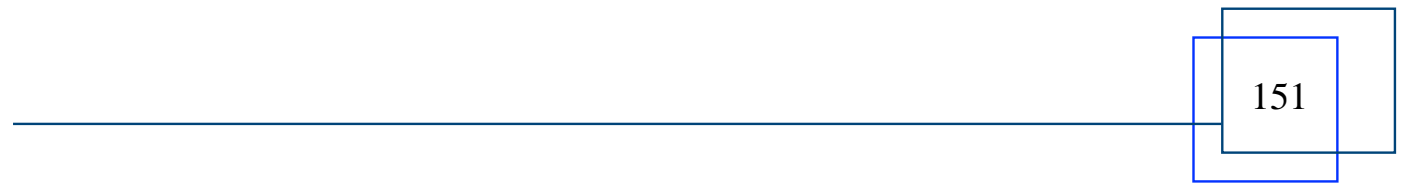




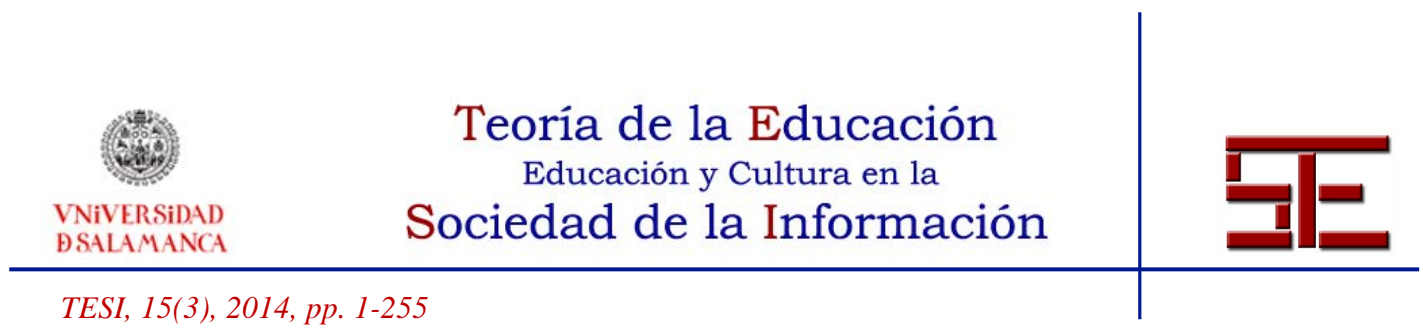

\section{OBJETO DE ESTUDIO}

\subsection{Pregunta de investigación}

Con base en los planteamientos anteriores se formuló la siguiente pregunta de investigación:

¿Se puede incidir en el desarrollo de las habilidades sociales de estos estudiantes de segundo grado de una preparatoria pública del Estado de Puebla, México, a través de actividades virtuales desarrolladas en la plataforma Moodle?

\subsection{Hipótesis de investigación}

A partir de esta pregunta se formuló la hipótesis de investigación que se presenta a continuación:

A través de actividades virtuales desarrolladas en la plataforma Moodle se logra incidir de manera positiva y significativa en las habilidades sociales de estudiantes de segundo grado de una preparatoria pública del Estado de Puebla, México.

\subsection{Definición de variables}

Variable independiente: Actividades virtuales para la promoción de las habilidades sociales: Conjunto de actividades de aprendizaje diseñadas en la plataforma Moodle encaminadas a incidir positivamente en las habilidades sociales.

Variable dependiente: Habilidades sociales: Destrezas para interactuar y relacionarse con otros, de manera eficaz y mutuamente satisfactoria.

\subsection{Objetivo}

El presente estudio tiene como objetivo implementar actividades virtuales en la plataforma Moodle para incidir de manera positiva y significativa en las habilidades sociales de estudiantes mexicanos de una preparatoria pública, de acuerdo al perfil de egreso de este nivel educativo y en respuesta a la necesidad de promover comportamientos pro-sociales en este grupo de estudiantes.

\subsection{Contexto}

Esta investigación se llevó a cabo en una preparatoria pública del Estado de Puebla,

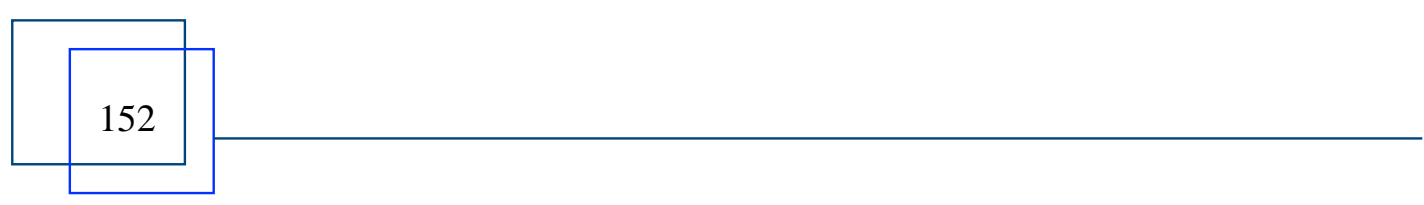




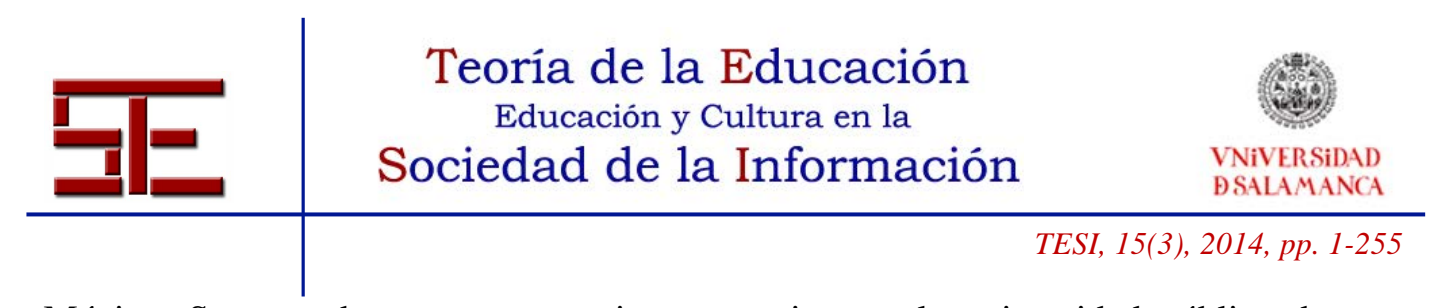

México. Se trata de una preparatoria perteneciente a la universidad pública de este Estado, ubicada en el centro histórico de la capital poblana.

Este plantel cuenta con una población de 300 alumnos en el segundo grado, los cuales están distribuidos en dos turnos: matutino y vespertino.

\subsection{Público-objetivo}

De la población estudiantil de segundo grado de la preparatoria en la que se realizó el presente estudio se obtuvo una muestra aleatoria. Esta muestra quedó conformada por 169 estudiantes, de los cuales 107 son mujeres y 62 son hombres. Las edades de los estudiantes seleccionados oscilan entre los 15 y los 17 años. La mayoría de ellos son originarios del Estado de Puebla (88\%) y el resto proviene de distintos estados de la República Mexicana.

100 sujetos de la muestra asisten al turno vespertino y 69 al turno matutino. Con base en una escala de calificaciones en la que el máximo puntaje es diez y el mínimo cinco, los promedios de calificaciones de los participantes oscilan entre seis y diez. Estos estudiantes se dedican de tiempo completo a las actividades escolares, es decir, no combinan sus estudios con alguna actividad laboral.

El nivel socioeconómico predominante de los participantes es el medio bajo. El 72\% de sus progenitores están casados. El $72 \%$ de los padres cuentan con estudios de licenciatura y el 58\% son empleados. En relación a las madres, el 31\% cuenta con una licenciatura y el $22 \%$ tiene estudios de preparatoria, el $46 \%$ se dedican a las labores del hogar y el 32\% son empleadas.

\subsection{Tipo de estudio}

Para la realización del presente estudio se adoptó un diseño cuasi-experimental pretestpostest de un solo grupo (Hernández, Fernández-Collado y Baptista, 2006; Martin, 2008; Bisquerra, 2009, y Navas, 2009). Este diseño de investigación consiste en realizar una comparación intra-grupo con mediciones antes y después del tratamiento (Martin, 2008). El diseño pretest-postest con un grupo consiste en que el investigador realiza una medición, llamada pretest o prueba diagnóstica, para establecer los niveles en los que se encuentra la variable objeto de estudio, después aplica un tratamiento o intervención y a continuación realiza una nueva medida, llamada postest o evaluación, para determinar el impacto que el tratamiento tuvo en la variable estudiada (Martin, 2008 y Bisquerra, 2009).

El resultado de este diseño es la valoración del cambio que se generó desde el pretest hasta el postest, mediado por el tratamiento (Bisquerra, 2009).

La ventaja de este tipo de diseño es contar con una referencia inicial, los resultados del

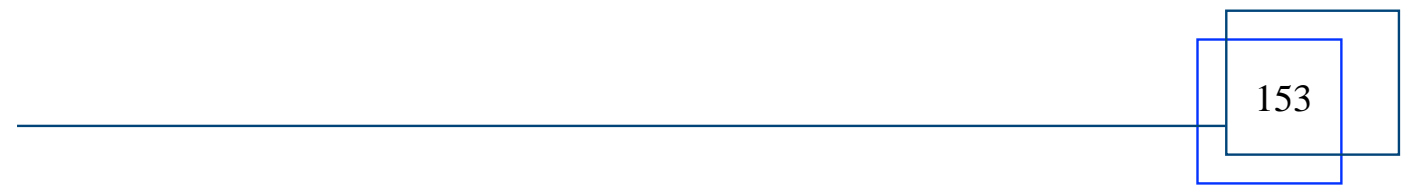




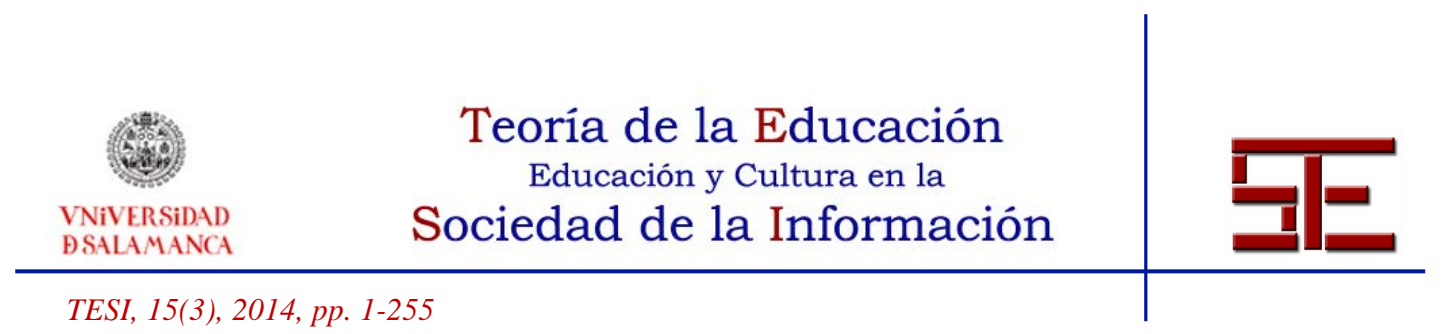

pretest, sobre los niveles del grupo en la variable dependiente antes del tratamiento, de modo que puedan compararse los resultados obtenidos después del tratamiento con esta primera referencia para determinar su nivel de impacto (Hernández et al., 2006).

\subsection{Instrumento}

El instrumento utilizado es la escala de habilidades sociales de Goldstein (Goldstein, McGinnis, Sprafkin, Gershaw y Klein, 1997, y Aguirre, 2004), adaptada para su aplicación electrónica. Esta escala evalúa las habilidades sociales básicas, habilidades sociales avanzadas, habilidades sociales relacionadas con el manejo de sentimientos y habilidades sociales alternativas.

La escala de habilidades sociales de Goldstein consta de 50 reactivos organizados por grupos de habilidades. Cada reactivo se responde de acuerdo a una escala del 1 al 5: "nunca utiliza bien la habilidad” (1), “casi nunca utiliza bien la habilidad” (2), “a veces utiliza bien la habilidad" (3), "casi siempre utiliza bien la habilidad" (4) y "siempre utiliza bien la habilidad” (5) (Aguirre, 2004).

Su calificación consiste en la sumatoria de las puntuaciones otorgadas por el sujeto a cada reactivo, por grupos de habilidad. La suma por grupo es catalogada en una escala del 1 al 5, donde uno equivale a muy bajo, dos a bajo, tres a medio, cuatro a alto y cinco a muy alto.

\section{REFERENTES TEÓRICOS}

El ser humano es social por naturaleza y es en esta tendencia a la sociabilidad o a la asociación con otras personas donde encuentra la satisfacción de sus necesidades de compañía, de convivencia y de ayuda mutua. El ser humano se hace con los otros, por los otros y para los otros, pues es en la relación con sus semejantes que se construye como persona.

El desarrollo social implica la adquisición de una serie de habilidades que le permiten al sujeto adaptarse al medio social, tanto en relación a lograr una autonomía e independencia personal que le permita desenvolverse por sí mismo de manera correcta y adecuada, como respecto a las relaciones interpersonales (Monjas y González, 1998).

En este contexto se entiende por habilidades sociales a las destrezas para interactuar y relacionarse con otros, de manera eficaz y mutuamente satisfactoria (Monjas y González, 1998). Estas habilidades permiten realizar la tarea interpersonal de manera competente.

Goldstein (Aguirre, 2004) organiza estas habilidades sociales en seis grandes grupos que incluyen aquellas capacidades y comportamientos que permiten a una persona

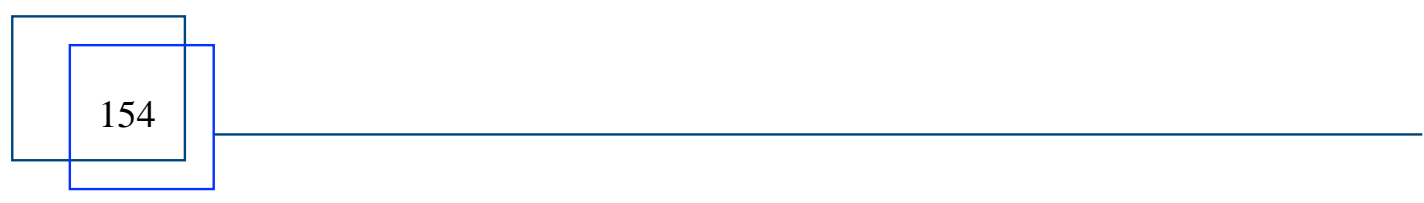




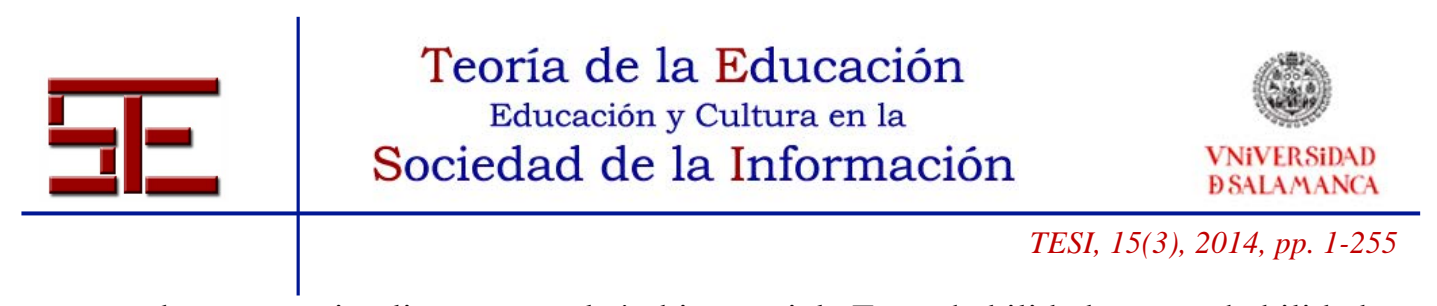

actuar de manera inteligente en el ámbito social. Estas habilidades son: habilidades sociales básicas, habilidades sociales avanzadas, habilidades sociales relacionadas con el manejo de sentimientos, habilidades alternativas, habilidades para hacer frente al estrés, y habilidades sociales de planificación.

Las habilidades sociales básicas, llamadas también primeras habilidades sociales, se refieren a las destrezas que permiten un desenvolvimiento social elemental o básico; aluden a los mínimos de cortesía convenidos en una sociedad. Incluyen aspectos básicos de comunicación, de establecimiento de los primeros vínculos interpersonales, identificación y expresión de los propios intereses así como de formas de mostrar agradecimiento por favores recibidos.

Estas habilidades implican:

- Escuchar y comprender la plática de otras personas.

- Tomar la iniciativa para entablar una conversación.

- Identificar los intereses de otros y empatarlos con los propios intereses.

- Solicitar información específica para realizar alguna acción o tarea.

- Agradecer los favores recibidos.

- Tomar la iniciativa para conocer nuevas personas.

- Promover el establecimiento de vínculos entre personas.

- Expresar los propios gustos y preferencias.

Las habilidades sociales avanzadas son las destrezas de desenvolvimiento social que implican una interacción más elaborada y la combinación de normas de comportamiento social. Refieren la capacidad para pedir ayuda, para integrarse a un grupo, para disculparse y para persuadir, para seguir instrucciones y para brindar explicaciones sobre tareas específicas.

Estas habilidades incluyen:

- Pedir ayuda.

- Integrarse a un grupo.

- Explicar una tarea específica.

- Entender y seguir instrucciones.

- Pedir disculpas por los errores cometidos.

- Persuadir.

Las habilidades relacionadas con los sentimientos son las destrezas relativas a un manejo adecuado de las propias emociones y a su expresión socialmente aceptada, de

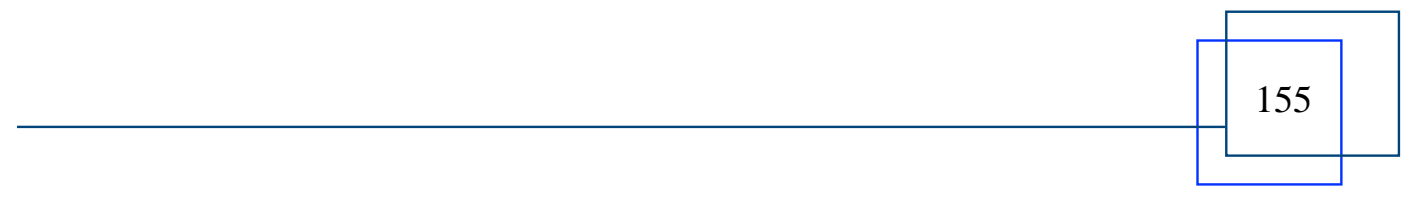




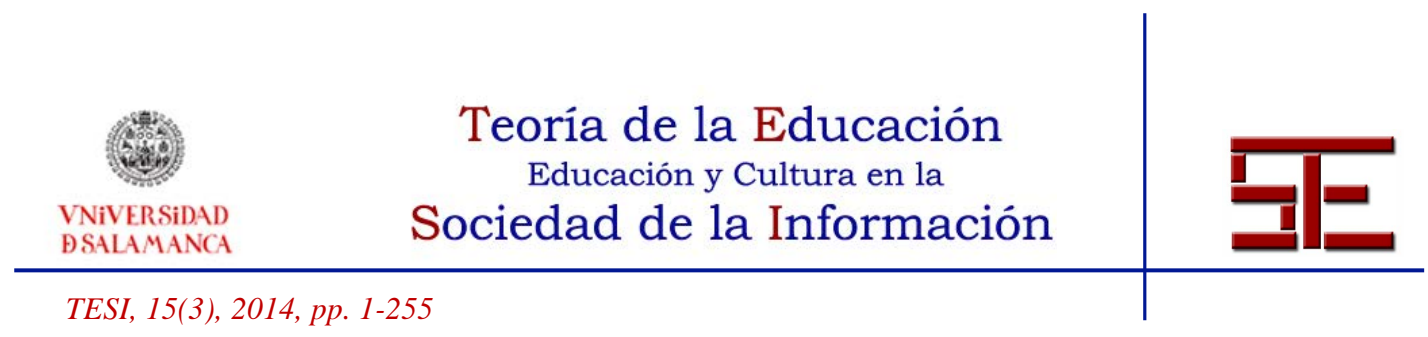

acuerdo a las normas de comportamiento de una determinada sociedad o grupo social. Aglutinan las capacidades para comprender, reconocer y expresar las propias emociones y las de los demás, preocuparse por otros, y recompensarse por una buena acción.

El manejo de estas habilidades hace posible que la persona:

- Comprenda y reconozca sus emociones.

- Exprese sus emociones.

- Comprenda las emociones de los demás.

- Comprenda el enfado de otras personas.

- Muestre interés o preocupación por otros.

- Reconozca y maneje sus propios miedos.

- Se recompense por realizar una buena acción.

Las habilidades sociales alternativas son las destrezas de interacción social que implican la búsqueda de vías diversas de abordaje para lograr el efecto deseado. Hacen referencia a la capacidad para compartir, ayudar, conciliar, defender los propios derechos, autocontrolarse, resolver conflictos y solicitar autorizaciones.

Comprenden lo siguiente:

- Capacidad para solicitar permiso a la persona indicada.

- Disposición a compartir.

- Disposición a ayudar.

- Capacidad para conciliar

- Capacidad de autocontrol.

- Capacidad para defender los propios derechos.

- Capacidad para controlarse cuando otros hacen bromas.

- Capacidad para mantenerse al margen de situaciones problemáticas.

- Capacidad para resolver conflictos sin pelear.

Las habilidades para hacer frente al estrés hacen referencia al modo como una persona puede enfrentar con ecuanimidad y mesura momentos de tensión, de contrariedad o de discrepancia, como son sentirse rechazado o apenado, enfrentar los propios errores o la injusticia, recibir críticas y reconocer el mérito de otros.

Estas capacidades implican:

156 


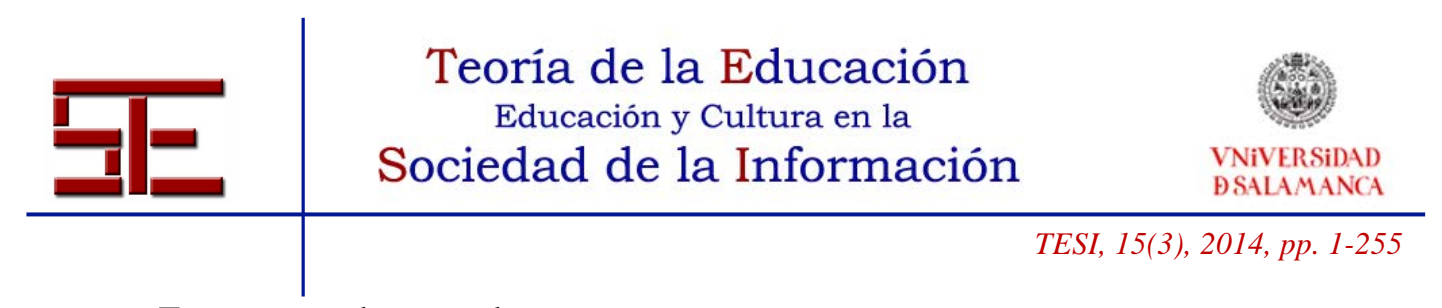

- Expresar su desacuerdo.

- Escuchar las críticas de otros y responderlas con imparcialidad.

- Halagar.

- Manejar la vergüenza y la timidez.

- Manejar el rechazo o el ser excluido.

- Expresar los propios sentimientos ante una injusticia.

- Analizar la propia posición y la de otro al tomar una decisión.

- Comprender los propios fracasos.

- Resolver confusiones generadas por un actuar incongruente.

- Comprender y manejar el ser acusado.

- Planificar una conversación problemática.

- Decidir qué hacer en una situación adversa.

Las habilidades de planificación implican la previsión para evitar problemas y dificultades, para optimizar el tiempo, para la resolución de problemas y para la toma de decisiones.

Estas habilidades hacen referencia a lo siguiente:

- Manejo del aburrimiento.

- Identificación de las causas de un problema.

- Toma de decisiones realistas antes de iniciar una tarea.

- Determinación de la propia competencia para realizar una tarea.

- Identificación y búsqueda de información necesaria para una tarea.

- Priorización de problemas.

- Análisis de opciones o posibilidades para elegir la mejor.

- Concentración en una tarea o actividad.

En el presente estudio se abordaron las habilidades sociales básicas, las habilidades sociales avanzadas, las habilidades relacionadas con el manejo de sentimientos y las habilidades alternativas. También se calculó un índice global de habilidades sociales a partir del puntaje promedio obtenido en cada uno de los grupos de habilidades sociales mencionados en este párrafo.

Respecto al desarrollo de actividades virtuales en la plataforma Moodle para la promoción de las habilidades sociales, se encuentra que, de acuerdo a Morales, E., Díaz,

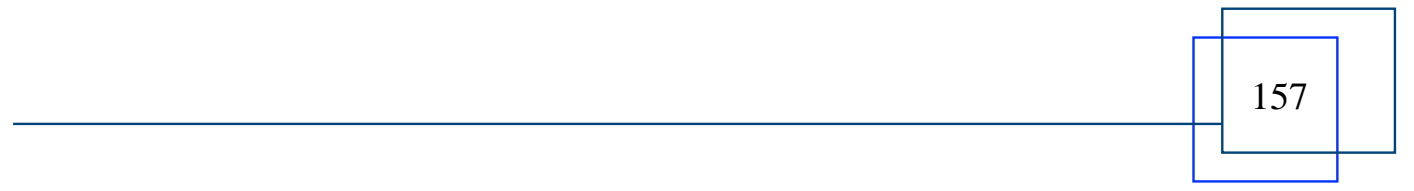




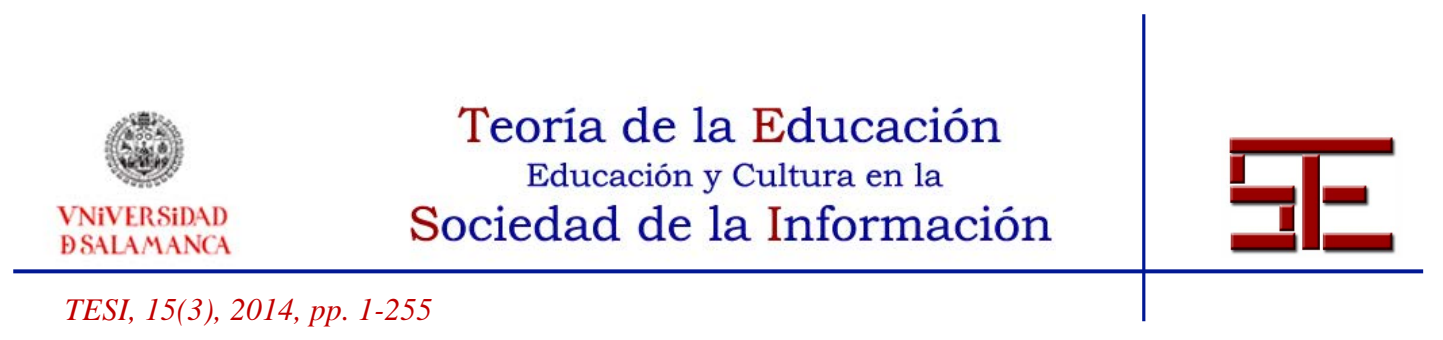

E. y García, F. (2011) el diseño de objetos de aprendizaje que involucren a los estudiantes en experiencias formativas significativas cobra gran relevancia en el contexto educativo actual, orientado al desarrollo de competencias que permitan el logro de altos estándares de desempeño profesional. De ahí que recomienden el diseño de objetos que contengan unidades mínimas de aprendizaje de modo que permitan un alto rango de usabilidad.

Por su parte, Cacheiro (2011) establece que los recursos de las tecnologías de información y comunicación contribuyen a los procesos de información, colaboración y aprendizaje, lo cual favorece la presentación de información relevante, el establecimiento de redes de intercambio y la consecución de conocimientos cognitivos, procedimentales y actitudinales.

Los aspectos planteados por estos autores brindan sustento al desarrollo de la propuesta se expone en este artículo.

\section{DESCRIPCIÓN}

Como se mencionó anteriormente, en este estudio se utilizó la escala de habilidades sociales de Goldstein con la intención de determinar los niveles de las habilidades sociales básicas, las habilidades sociales avanzadas, las habilidades relacionadas con el manejo de sentimientos y las habilidades sociales alternativas de los participantes antes de la aplicación de las actividades virtuales orientadas al desarrollo de estas habilidades, diseñadas en la plataforma Moodle.

Con base en la información obtenida mediante la referida escala, se procedió a la realización del diseño instruccional del curso tomando como base la metodología propuesta por Gloria Yukavetsky (2003) y Alvarado (2003). Estos autores establecen cinco pasos para desarrollar un diseño instruccional en ambientes virtuales de aprendizaje. Estos pasos son: análisis del contexto, diseño, desarrollo, implantación e implementación, y evaluación.

A continuación se reporta el diseño instruccional de las actividades virtuales encaminadas a la promoción de las habilidades sociales de los participantes:

El primer paso, denominado análisis del contexto, se llevó a cabo con base en la información aportada por los profesores, directivos y padres de familia de los participantes. También se consideró el perfil de egreso y el plan de estudios de la preparatoria, las especificaciones de operación del aula virtual en Moodle de la institución, y los resultados obtenidos, en la primera aplicación, en la escala de habilidades sociales de Goldstein.

Los siguientes pasos, que son el diseño, el desarrollo, la implantación e

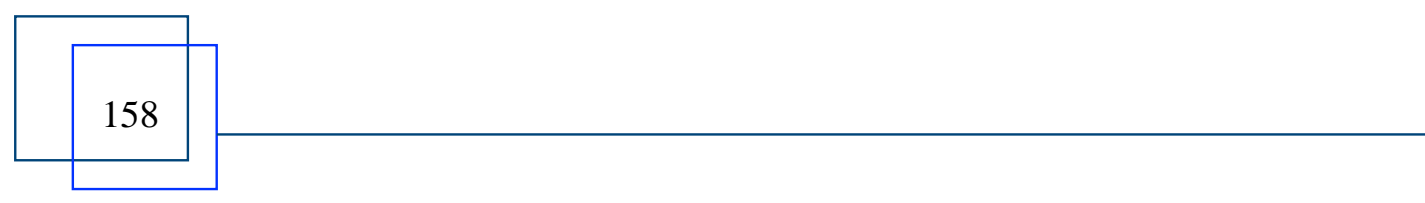




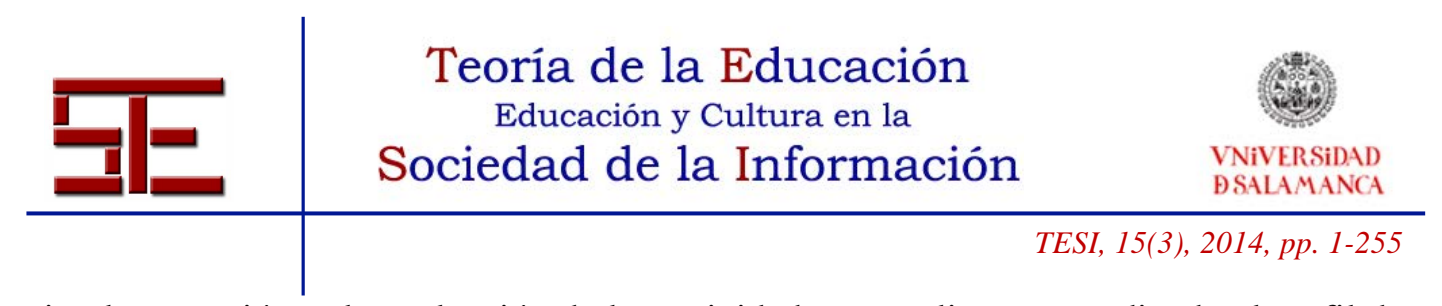

implementación, y la evaluación de las actividades se realizaron atendiendo al perfil de los participantes y a las características del aula virtual en la plataforma Moodle de la preparatoria.

Las sesiones virtuales fueron diseñadas de modo que fueran auto-dirigidas, es decir, que permitan que los participantes puedan desarrollarlas sin necesidad de contar con la guía de un tutor. Desde esta perspectiva el tutor tiene como tarea principal enviar a los estudiantes comentarios encaminados a reconocer e impulsar la participación de los mismos, además de revisar y retroalimentar las actividades ya realizadas por los participantes.

Los recursos de Moodle que fueron empleados para el diseño de las actividades virtuales orientadas al desarrollo de habilidades sociales son: etiquetas, cuestionarios y foros. También se insertaron presentaciones gráficas sobre los contenidos abordados.

De acuerdo a la catalogación de unidades independientes según un nivel de conocimiento específico propuesta por Morales, Díaz y García (2011) para los objetos de aprendizaje, se puede considerar que estas actividades son del tipo reflexión y actitud (TC_RA) que hacen referencia a los objetos de aprendizaje que promueven el desarrollo de actitudes valores y normas mediante la reflexión y la crítica y cuyas palabras clave son: reflexión actitud, toma de decisiones, valores y normas (p. 111).

Las actividades tuvieron como objetivo que el participante fuera capaz de incorporar en su actuar habilidades sociales que le permitan entablar relaciones interpersonales sanas y equilibradas.

Para alcanzar este objetivo, se desarrollaron las actividades virtuales que se enuncian a continuación:

- Frase de sensibilización encaminada a introducir al participante en el tema de la comunicación asertiva.

- Video sobre la importancia de ser agradecido, considerado como parte de las habilidades sociales básicas.

- Redacción de una carta de agradecimiento a la propia familia, dirigida al desarrollo de las habilidades para el manejo de sentimientos.

- Lectura sobre la comunicación asertiva que brinda elementos para desarrollar la habilidad de comunicación honesta, oportuna y respetuosa en la que se preservan los propios derechos y los de los demás.

- Actividad interactiva sobre el lenguaje no verbal para que aprendieran a descifrar los diversos mensajes que se transmiten en este tipo de comunicación.

- Actividad interactiva sobre la falta de comunicación y su relación con la generación de conflictos interpersonales.

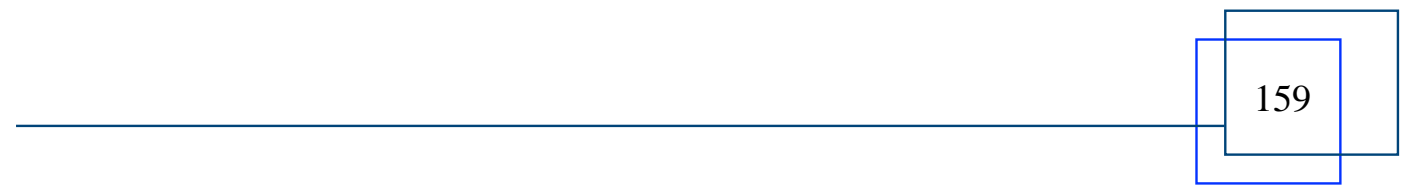




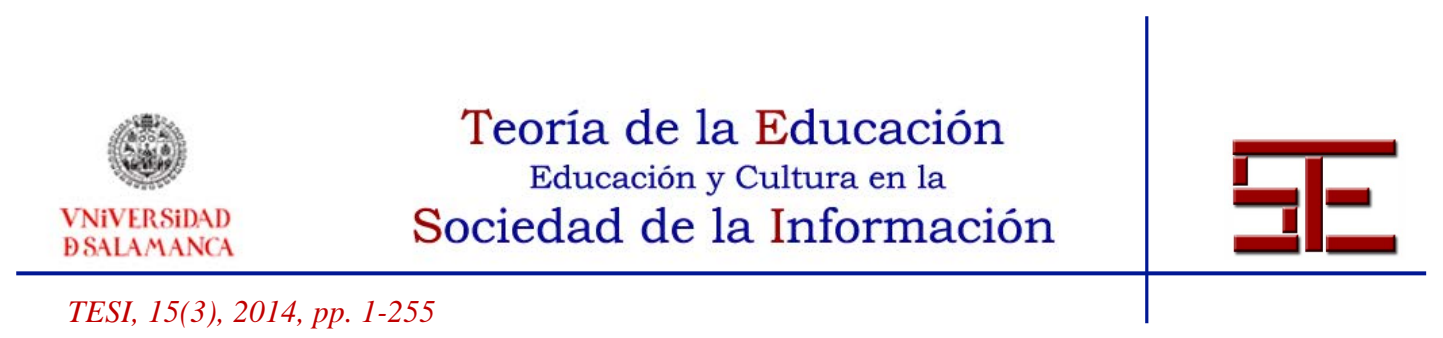

- Video sobre la importancia que tiene la comunicación en las relaciones interpersonales.

- Frase de sensibilización en torno a la realización de acciones sencillas para promover la colaboración y la armonía con los demás.

- Lectura sobre las diferencias e implicaciones personales e interpersonales del compañerismo y la amistad en el contexto escolar y social.

- Página de la amistad para promover la creación de redes de ayuda mutua entre los estudiantes de segundo grado de la preparatoria, turnos matutino y vespertino.

- Video sobre ayuda mutua y cooperación, su importancia e impacto en la construcción de sociedades justas y armoniosas.

- Cuestionario "ayudo cuando..." para reafirmar acciones de apoyo y cooperación.

- Video sobre altruismo para concientizar a los estudiantes sobre la importancia de ayudar a personas en condiciones de vida poco favorables para cubrir sus necesidades básicas.

- Foro sobre momentos de altruismo y participación que los estudiantes han vivido en la vida cotidiana, encaminado a favorecer el conocimiento mutuo y al reconocer que en la vida ordinaria es posible ayudar a los demás y participar en las decisiones y acciones.

- Frase de cierre para reafirmar la importancia de compartir.

Los estudiantes llevaron a cabo estas actividades en un lapso de seis semanas, con dos horas de trabajo a la semana. Las actividades se realizaron en el marco de la materia Laboratorio de Computación, pues es en el marco de esta clase que los alumnos tienen acceso al uso de los ordenadores dentro del horario escolar. Durante la realización de las actividades, los estudiantes contaron con el apoyo, de manera presencial, del docente de la materia en relación a cuestiones técnicas, y de un tutor virtual respecto a la realización de las actividades en sí mismas, con el cual pudieron entrar en comunicación tanto de manera sincrónica como asincrónica.

Finalmente, se aplicó la escala de habilidades sociales de Goldstein para evaluar el impacto que estas actividades tuvieron en las habilidades sociales de los participantes. También se utilizó la encuesta en línea sobre ambiente constructivista de aprendizaje (Colles-Real) proporcionada por la plataforma Moodle para que los participantes evaluaran las actividades.

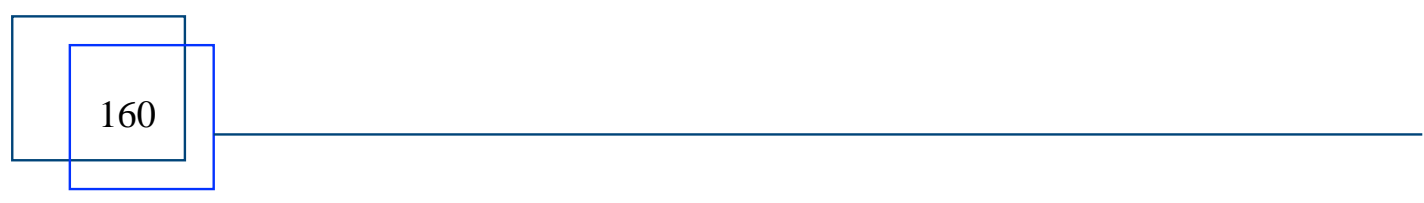




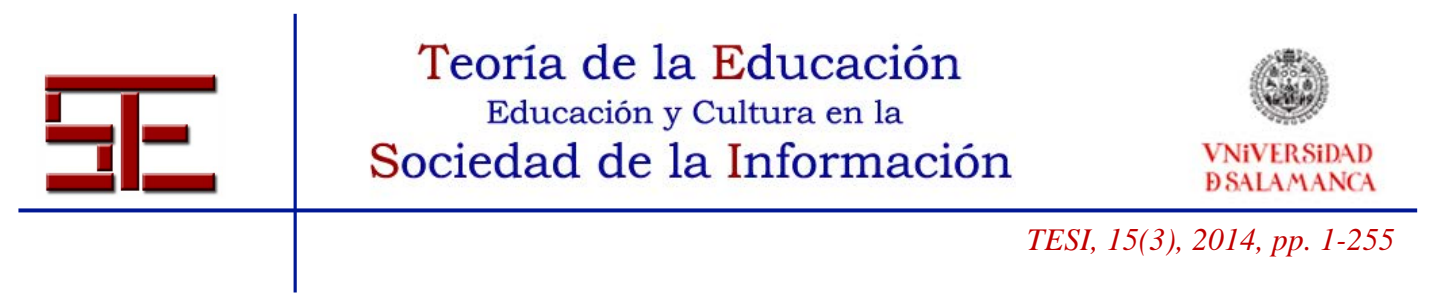

\section{RESULTADOS}

Con base en los datos obtenidos en la aplicación previa y posterior a la implementación de las actividades virtuales de la escala de habilidades sociales de Goldstein, se realizaron pruebas estadísticas para cada grupo de las habilidades evaluadas, con la intención de establecer si las actividades propuestas incidieron en el mejoramiento de las habilidades sociales de los participantes. En los siguientes apartados se presenta el análisis de los resultados obtenidos mediante las pruebas estadísticas aplicadas.

\subsection{Análisis estadístico de las habilidades sociales básicas}

Las habilidades sociales básicas refieren aspectos elementales de la comunicación como son: entablar los primeros vínculos interpersonales, identificar y expresar intereses, y agradecer favores recibidos (Goldstein et al., 1997 y Aguirre, 2004).

La escala de habilidades sociales de Goldstein arrojó, en la aplicación previa al tratamiento, los siguientes resultados: un sujeto (1\%) se ubicó en el nivel bajo, dos sujetos (1\%) se ubicaron en el nivel medio bajo, 30 sujetos (18\%) en el nivel medio, 99 sujetos (58\%) en el medio alto y 37 sujetos (22\%) en el nivel alto. La media de las puntuaciones fue de 31.4 y la desviación estándar de 5.

En la aplicación posterior al tratamiento se obtuvieron los siguientes resultados: ningún sujeto se ubicó en el nivel bajo, un sujeto (1\%) se ubicó en el medio bajo, 23 sujetos (13\%) en el nivel medio, 105 sujetos (62\%) en el nivel medio alto y 40 sujetos (24\%) en el nivel alto. La media de las puntuaciones posteriores a la aplicación de tratamiento fue de 32.1 y la desviación estándar de 4.7.

Tabla 1. Distribución de frecuencias de las habilidades sociales básicas.

Pretest-Postest

\begin{tabular}{|l|l|l|l|l|}
\hline \multicolumn{2}{|l|}{ Habilidades sociales básicas } \\
\hline \multirow{2}{*}{ Nivel } & \multicolumn{2}{l|}{ Pretest } & \multicolumn{2}{l|}{ Postest } \\
\cline { 2 - 5 } & (f) & $(\%)$ & (f) & $(\%)$ \\
\hline Bajo & 1 & $1 \%$ & 0 & $0 \%$ \\
\hline Medio-bajo & 2 & $1 \%$ & 1 & $1 \%$ \\
\hline Medio & 30 & $18 \%$ & 23 & $13 \%$ \\
\hline
\end{tabular}

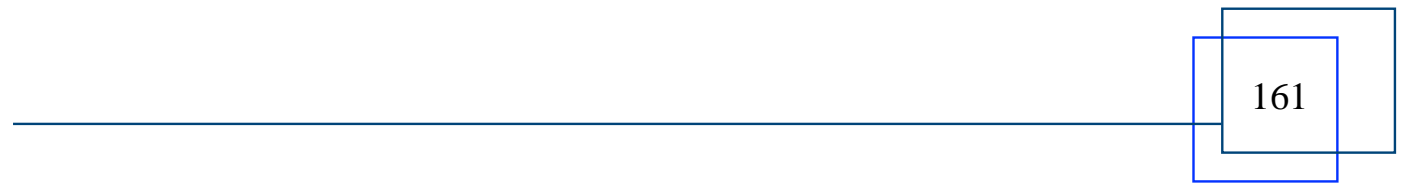




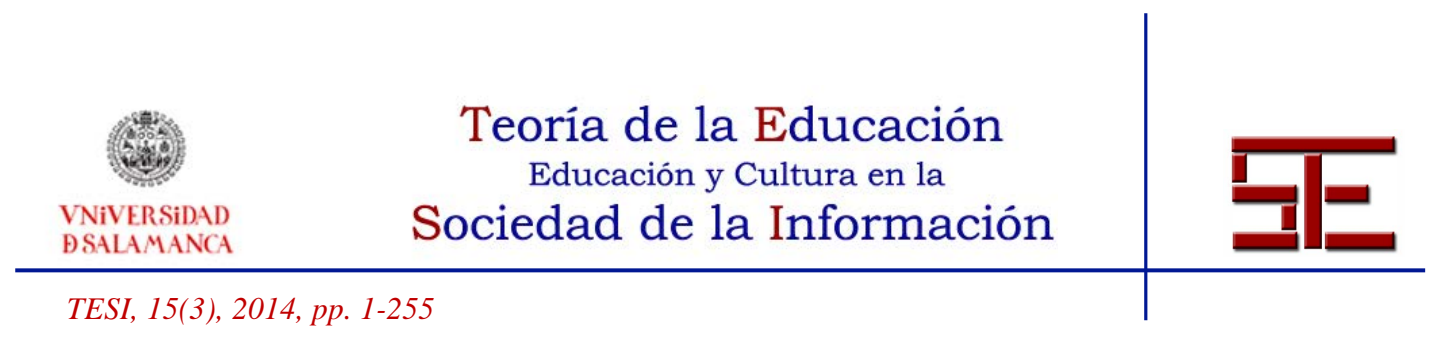

\begin{tabular}{|l|l|l|l|l|}
\hline Medio-alto & 99 & $58 \%$ & 105 & $62 \%$ \\
\hline Alto & 37 & $22 \%$ & 40 & $24 \%$ \\
\hline Total & $\mathbf{1 6 9}$ & $\mathbf{1 0 0 \%}$ & $\mathbf{1 6 9}$ & $\mathbf{1 0 0 \%}$ \\
\hline Media & 31.4 & & 32.1 & \\
\hline $\begin{array}{l}\text { Desviación } \\
\text { estándar }\end{array}$ & 5 & 4.7 & \\
\hline
\end{tabular}

Con la intención de establecer si las actividades virtuales incidieron de manera significativa en las habilidades sociales básicas de los participantes, se aplicó la prueba t de Student con un nivel de significación de 0.01, cuyo puntaje teórico es de 2.6. El nivel determinado por la prueba (“t” empírica) es de 1.6, lo que se descarta un incremento significativo en los puntajes obtenidos en la escala de habilidades sociales de Goldstein en relación a las habilidades sociales básicas tras el tratamiento.

\subsection{Análisis estadístico de las habilidades sociales avanzadas}

Las habilidades sociales avanzadas aluden a las acciones necesarias para pedir ayuda, integrarse a un grupo, disculparse y persuadir, seguir instrucciones y brindar explicaciones sobre tareas específicas (Goldstein et al., 1997 y Aguirre, 2004).

En la aplicación de la escala de habilidades sociales de Goldstein antes del tratamiento, 49 sujetos (29\%) se ubicaron en el nivel bajo, un sujeto (1\%) se ubicó en el nivel medio bajo, 34 sujetos (20\%) se ubicaron en el nivel medio, 77 sujetos (45\%) se ubicaron en el nivel medio alto y 8 sujetos (5\%) se ubicaron en el nivel alto. La media de las puntuaciones fue de 17.3 y la desviación estándar fue de 7.7.

En el postest, un sujeto (1\%) se ubicó en el nivel bajo, dos sujetos (1\%) se ubicaron en el nivel medio bajo, 20 sujetos (12\%) alcanzaron el nivel medio, 83 sujetos (49\%) se ubicaron en el medio alto y 63 sujetos (37\%) se ubicaron en el nivel alto. La media fue de 24.7 y la desviación estándar de 4.

Tabla 2. Distribución de frecuencias de las habilidades sociales avanzadas.

Pretest-Postest

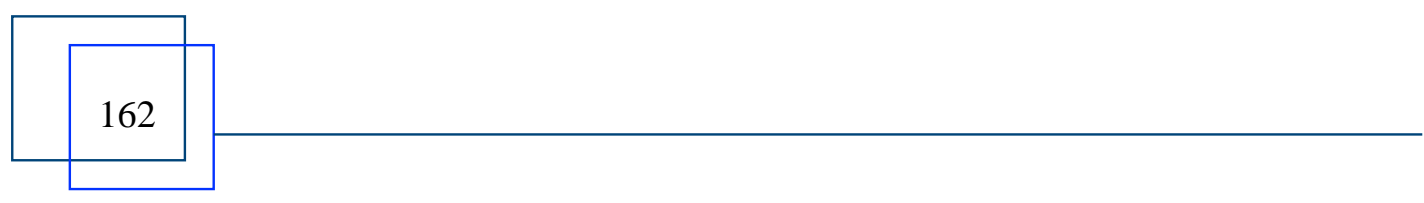




\begin{tabular}{|c|c|c|c|c|}
\hline \multicolumn{5}{|c|}{$\begin{array}{c}\text { Teoría de la Educación } \\
\text { Educación y Cultura en la } \\
\text { Sociedad de la Información }\end{array}$} \\
\hline \multicolumn{5}{|r|}{$\overline{T E S I, 1}$} \\
\hline \multicolumn{5}{|c|}{ Habilidades sociales avanzadas } \\
\hline \multirow{2}{*}{ Nivel } & \multicolumn{2}{|c|}{ Pretest } & \multicolumn{2}{|c|}{ Postest } \\
\hline & (f) & $(\%)$ & (f) & $(\%)$ \\
\hline Bajo & 49 & $29 \%$ & 1 & $1 \%$ \\
\hline Medio-bajo & 1 & $1 \%$ & 2 & $1 \%$ \\
\hline Medio & 34 & $20 \%$ & 20 & $12 \%$ \\
\hline Medio-alto & 77 & $45 \%$ & 83 & $49 \%$ \\
\hline Alto & 8 & $5 \%$ & 63 & $37 \%$ \\
\hline Total & 16 & $100 \%$ & 169 & $100 \%$ \\
\hline Media & \multicolumn{2}{|c|}{17.3} & \multicolumn{2}{|c|}{24.7} \\
\hline $\begin{array}{l}\text { Desviación } \\
\text { estándar }\end{array}$ & \multicolumn{2}{|c|}{7.7} & \multicolumn{2}{|l|}{4} \\
\hline
\end{tabular}

Para establecer si se logró una diferencia significativa en estas habilidades mediante la realización de las actividades virtuales, se aplicó la prueba t de Student con un nivel de significación de 0.01, cuyo puntaje teórico es de 2.6. El nivel determinado por la prueba (“t” empírica) es de 10.89, lo que indica que se logró un incremento significativo en los puntajes obtenidos en la escala de habilidades sociales de Goldstein en relación a las habilidades sociales avanzadas, después del tratamiento.

\subsection{Análisis estadístico de las habilidades para el manejo de sentimientos}

Las habilidades para el manejo de sentimientos están encaminadas a comprender, reconocer y expresar las propias emociones y las de los demás, preocuparse por otras personas, y recompensarse a sí mismo por haber realizado una buena acción (Goldstein et al. 1997 y Aguirre, 2004).

En el pretest se identificaron seis sujetos (4\%) en el nivel bajo, dos sujetos (1\%) en el nivel medio bajo, 20 sujetos (12\%) en el nivel medio, 71 sujetos (42\%) en el nivel medio alto y 70 sujetos (41\%) en el nivel alto. La media de las puntuaciones fue de 24.6 y la desviación estándar fue de 5.4.

En el postest, ningún sujeto se ubicó en el nivel bajo, un sujeto (1\%) en el nivel medio

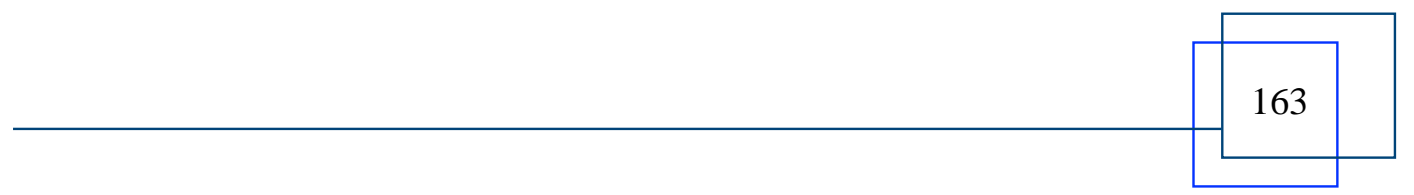




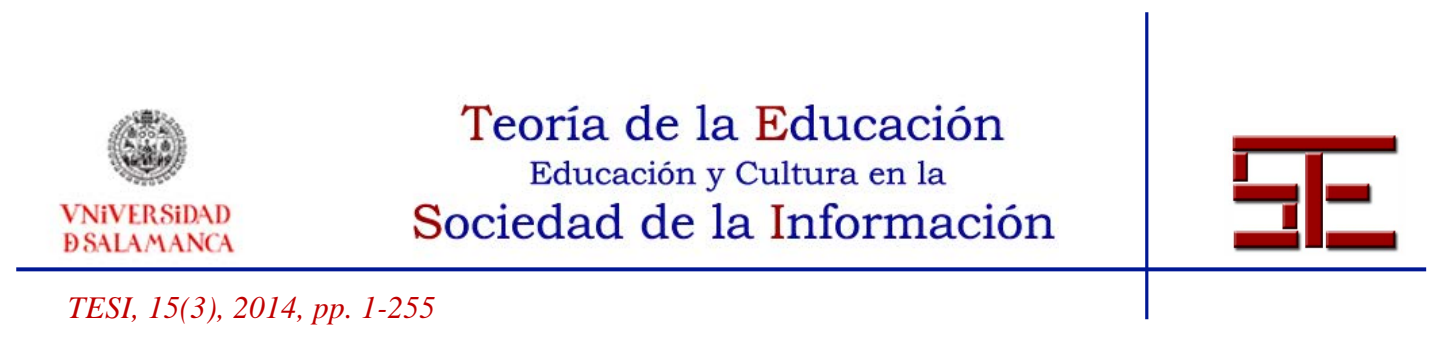

bajo, 12 sujetos (7\%) en el nivel medio, 47 sujetos (28\%) en el medio alto y 109 sujetos (64\%) en el alto. La media de las puntuaciones fue de 26.7 y la desviación estándar de 3.8.

Tabla 3. Distribución de frecuencias de las habilidades para el manejo de sentimientos.

Pretest-Postest

\begin{tabular}{|l|l|l|l|l|}
\hline \multirow{2}{*}{ Habilidades para el manejo de sentimientos } \\
\hline \multirow{2}{*}{ Nivel } & \multicolumn{2}{|l|}{ Pretest } & \multicolumn{2}{l|}{ Postest } \\
\cline { 2 - 5 } & (f) & $(\%)$ & $(\mathrm{f})$ & $(\%)$ \\
\hline Bajo & 6 & $4 \%$ & 0 & $0 \%$ \\
\hline Medio-bajo & 2 & $1 \%$ & 1 & $1 \%$ \\
\hline Medio & 20 & $12 \%$ & 12 & $7 \%$ \\
\hline Medio-alto & 71 & $42 \%$ & 47 & $28 \%$ \\
\hline Alto & 70 & $41 \%$ & 109 & $64 \%$ \\
\hline Total & $\mathbf{1 6 9}$ & $\mathbf{1 0 0 \%}$ & $\mathbf{1 6 9}$ & $\mathbf{1 0 0 \%}$ \\
\hline Media & 24.6 & & 26.7 & \\
\hline $\begin{array}{l}\text { Desviación } \\
\text { estándar }\end{array}$ & 5.4 & & 3.8 & \\
\hline
\end{tabular}

Con la intención de establecer si se logró una diferencia significativa tras la realización de las actividades, se aplicó la prueba t de Student con un nivel de significación de 0.01, y un puntaje teórico de 2.6. El nivel determinado por la prueba (“t” empírica) es de 4.48, lo que indica que se logró un incremento significativo en los puntajes obtenidos mediante la escala de habilidades sociales de Goldstein en las habilidades para el manejo de sentimientos, después del tratamiento.

\subsection{Análisis estadístico de las habilidades sociales alternativas}

Las habilidades sociales alternativas están enfocadas a compartir, ayudar, conciliar, defender los propios derechos, auto-controlarse, resolver conflictos y solicitar

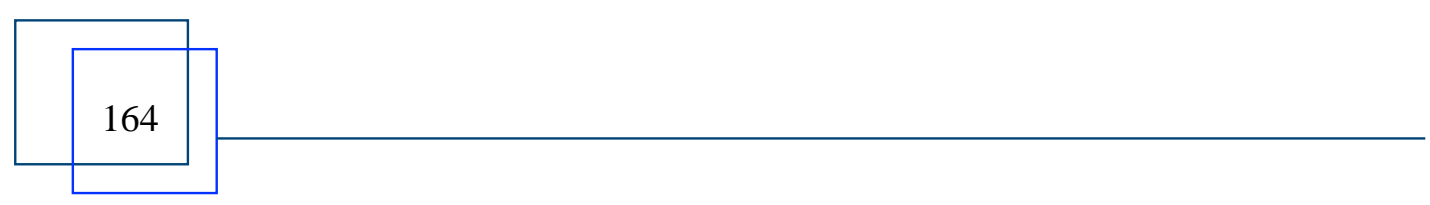




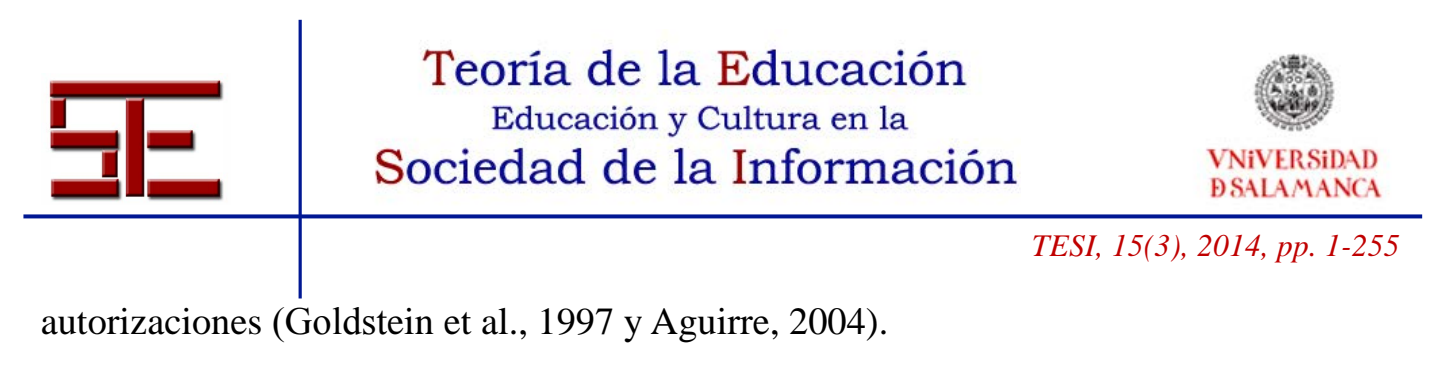

En la aplicación previa de la escala de habilidades sociales de Goldstein se encontró que 20 sujetos (12\%) se ubicaron en el nivel bajo, 8 sujetos (5\%) se ubicaron en el nivel medio bajo, 62 sujetos (36\%) en el nivel medio, 79 sujetos (47\%) en el medio alto y ningún sujeto en el alto. La media de las puntuaciones fue de 31.4 y la desviación estándar de 9.3.

En la aplicación posterior al tratamiento se obtuvieron los siguientes resultados: ningún sujeto se ubicó en el nivel bajo, 5 sujetos (3\%) se ubicaron en el medio bajo, 58 sujetos (34\%) en el nivel medio, 95 sujetos (56\%) en el nivel medio alto y 11 sujetos (7\%) en el nivel alto. La media de las puntuaciones fue de 36.1 y la desviación estándar de 5.2.

Tabla 4. Distribución de frecuencias de las habilidades sociales alternativas.

Pretest-Postest

\begin{tabular}{|l|l|l|l|l|}
\hline \multirow{4}{*}{ Habilidades sociales alternativas } \\
\hline \multirow{2}{*}{ Nivel } & \multicolumn{2}{l|}{ Pretest } & \multicolumn{2}{l|}{ Postest } \\
\cline { 2 - 5 } & (f) & $(\%)$ & (f) & $(\%)$ \\
\hline Bajo & 20 & $12 \%$ & 0 & $0 \%$ \\
\hline Medio-bajo & 8 & $5 \%$ & 5 & $3 \%$ \\
\hline Medio & 62 & $36 \%$ & 58 & $34 \%$ \\
\hline Medio-alto & 79 & $47 \%$ & 95 & $56 \%$ \\
\hline Alto & 0 & $0 \%$ & 11 & $7 \%$ \\
\hline Total & $\mathbf{1 6 9}$ & $\mathbf{1 0 0 \%}$ & $\mathbf{1 6 9}$ & $\mathbf{1 0 0 \%}$ \\
\hline Media & 31.4 & & 36.1 & \\
\hline $\begin{array}{l}\text { Desviación } \\
\text { estándar }\end{array}$ & 9.3 & & 5.2 & \\
\hline
\end{tabular}

Con la intención de establecer si se logró una diferencia significativa tras la realización del tratamiento, se aplicó la prueba t de Student con un nivel de significación de 0.01 , cuyo puntaje teórico es de 2.6. El nivel determinado por la prueba (“t” empírica) es de 5.77 , lo que verifica un incremento significativo en los puntajes obtenidos en la escala

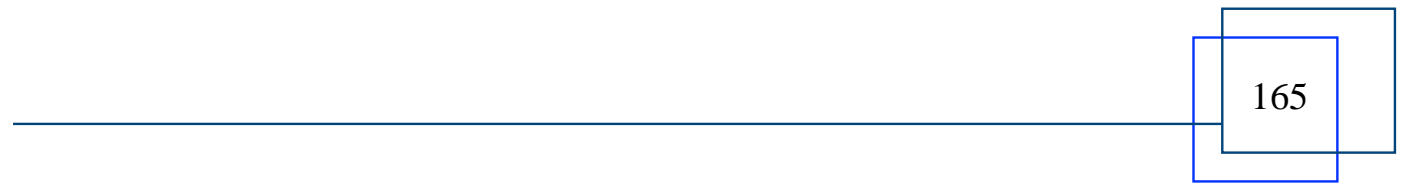




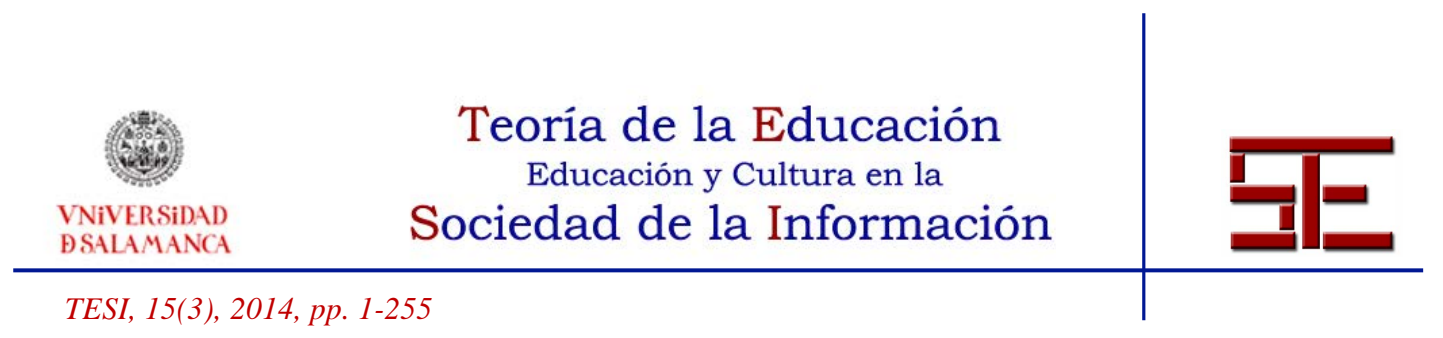

de habilidades sociales de Goldstein en relación a las habilidades sociales alternativas, después de la aplicación de las actividades virtuales.

\subsection{Factor global de las habilidades sociales}

El factor global de las habilidades sociales se obtiene del promedio de los cuatro grupos de habilidades evaluados a través de la escala de habilidades sociales de Goldstein, a saber: habilidades sociales básicas, habilidades sociales avanzadas, habilidades para el manejo de sentimientos y habilidades sociales alternativas.

Al calcular el promedio de las puntuaciones obtenidas de los cuatro grupos de habilidades evaluados para determinar el puntaje global, en el pretest se obtuvo lo siguiente: 0 sujetos ( $0 \%$ ) se ubicaron en el nivel bajo, 7 sujetos (4\%) en el nivel medio bajo, 71 sujetos (42\%) en el nivel medio, 87 sujetos (52\%) en el nivel medio alto y 4 sujetos (2\%) en el nivel alto. La media de las puntuaciones fue de 104.7 y la desviación estándar de 16.8.

Las puntuaciones obtenidas en el postest indican que 0 sujetos ( $0 \%)$ se ubicaron en el nivel bajo, 2 sujetos (1\%) en el medio bajo, 20 sujetos (12\%) en el nivel medio, 119 sujetos (70\%) en el nivel medio alto y 28 sujetos (17\%) en el nivel alto. La media de las puntuaciones fue de 120 y la desviación estándar de 16.

Tabla 5. Distribución de frecuencias del factor global de las habilidades sociales.

Pretest-Postest

\begin{tabular}{|l|l|l|l|l|}
\hline \multirow{2}{*}{ Factor Global de Habilidades Sociales } \\
\hline \multirow{2}{*}{ Nivel } & \multicolumn{2}{l|}{ Pretest } & \multicolumn{2}{l|}{ Postest } \\
\cline { 2 - 5 } & (f) & $(\%)$ & (f) & $(\%)$ \\
\hline Bajo & 0 & $0 \%$ & 0 & $0 \%$ \\
\hline Medio-bajo & 7 & $4 \%$ & 2 & $1 \%$ \\
\hline Medio & 71 & $42 \%$ & 20 & $12 \%$ \\
\hline Medio-alto & 87 & $52 \%$ & 119 & $70 \%$ \\
\hline Alto & 4 & $2 \%$ & 28 & $17 \%$ \\
\hline Total & $\mathbf{1 6 9}$ & $\mathbf{1 0 0 \%}$ & $\mathbf{1 6 9}$ & $\mathbf{1 0 0 \%}$ \\
\hline
\end{tabular}

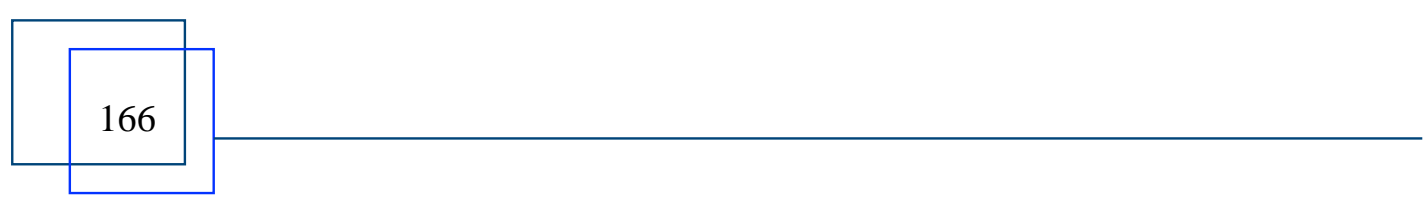




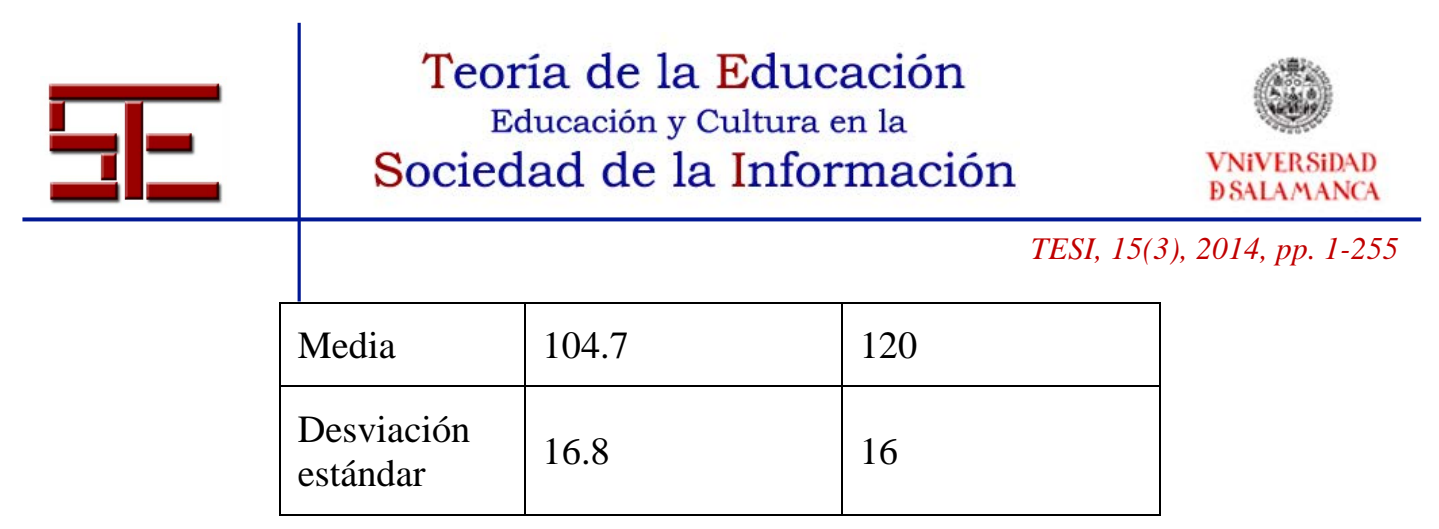

Mediante el uso de la prueba t de Student se pudo verificar que con las actividades virtuales desarrolladas en la plataforma Moodle se logró una mejora significativa de las habilidades sociales consideradas de manera global, al arrojar un puntaje (“t” empírica) de 8.98, con un nivel de significación de 0.01 que indica una "t" teórica de 2.6.

Como se mencionó, para evaluar las actividades virtuales diseñadas en la plataforma Moodle, se aplicó la encuesta en línea sobre ambiente constructivista de aprendizaje (Colles-Real) para que los participantes las calificaran a partir de la siguiente escala: 5 (excelente), 4 (muy bien), 3 (bien), 2 (regular), 1 (mal). La calificación promedio obtenida para cada aspecto evaluado por la encuesta Colles-Real es la siguiente:

- Relevancia del curso: se define como el interés que las actividades virtuales despertaron en los participantes y el impacto que las mismas tuvieron en su práctica. Su calificación promedio es de 4 (muy bien).

- Pensamiento reflexivo: hace referencia a la promoción, a través de las actividades virtuales, de una postura crítica ante el propio aprendizaje, las propias ideas, las aportaciones de los compañeros y hacia las lecturas. Fue calificado con 4 (muy bien).

- Interactividad: alude al intercambio de opiniones y aportaciones entre los participantes y el número de comentarios recibidos por cada uno de ellos. Obtuvo la calificación de 3.4 (bien).

- Apoyo del tutor: evalúa la actuación del tutor en relación a aspectos tales como estimular la reflexión, animar a los participantes y ejemplificar las disertaciones. Su calificación fue de 3.7 (muy bien).

- Apoyo de compañeros: evalúa si el usuario se sintió animado, elogiado y valorado por sus compañeros y si a través de las interacciones se logró generar un ambiente de empatía. Recibió una calificación de 3.3 (bien).

- Interpretación: en este rubro el usuario evalúa si hubo entendimiento entre los participantes y con el tutor. Alcanzó una calificación de 3.8 (muy bien).

En la evaluación cualitativa que la misma encuesta ofrece, los participantes expresaron que se sintieron beneficiados con las actividades y que estas les parecieron pertinentes para el desarrollo de sus habilidades sociales. 


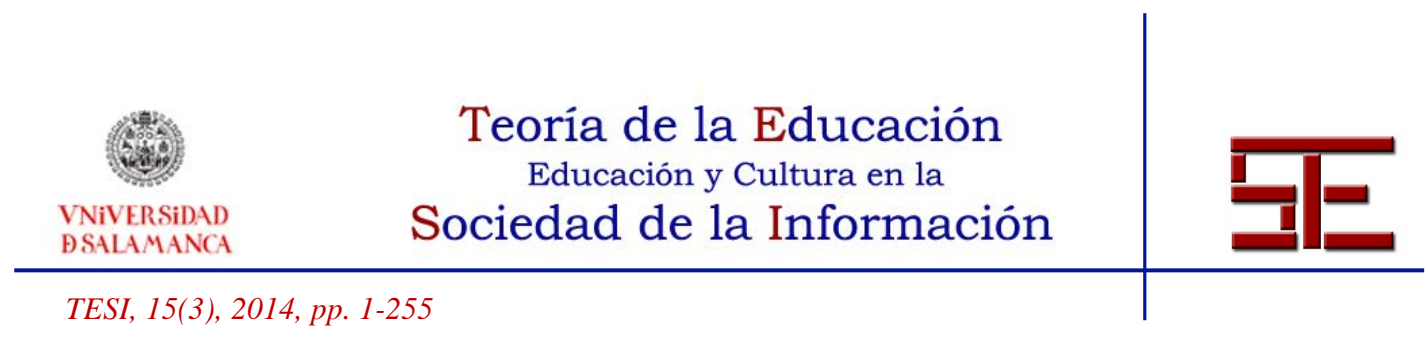

\section{CONCLUSIONES}

Este estudio tuvo como objetivo determinar la incidencia de actividades virtuales diseñadas en la plataforma Moodle sobre las habilidades sociales de estudiantes de segundo grado de una preparatoria pública del Estado de Puebla, México.

El análisis estadístico de los puntajes obtenidos mediante la escala de habilidades sociales de Goldstein permite afirmar que las actividades virtuales implementadas tuvieron un impacto positivo y significativo en las destrezas de los participantes para entablar relaciones interpersonales sanas y equilibradas. Se considera que esta incidencia del tratamiento sobre las habilidades sociales de los estudiantes de segundo grado de la preparatoria en la que se realizó el estudio contribuye a elevar el perfil de los egresados de este nivel educativo y puede llevar a la mejora de su vida social.

En concreto, se logró que los participantes a través de estas actividades virtuales:

- Mantuvieran sus niveles de desempeño en las habilidades sociales básicas.

- Mejoraran sus niveles de desempeño en las habilidades sociales avanzadas.

- Mejoraran sus habilidades relacionadas con el manejo de sentimientos.

- Mejoraran sus habilidades sociales alternativas.

- En suma, mejoraran sus habilidades sociales de manera global, pues vieron favorecido el desarrollo conjunto de sus capacidades para entablar relaciones interpersonales.

A partir de estos resultados se puede considerar que las tecnologías aplicadas a la educación y en particular las plataformas virtuales de aprendizaje proveen de recursos valiosos para promover el desarrollo de habilidades y destrezas, en este caso circunscritas al ámbito de las relaciones interpersonales, vinculadas a los perfiles de egreso escolar, que en el presente estudio aludieron a las competencias sociales a desarrollar en el nivel bachillerato.

La promoción de habilidades sociales mediante actividades virtuales diseñadas en la plataforma Moodle puede beneficiar a los estudiantes en su desenvolvimiento social y en las relaciones que entablen con sus familiares, profesores, compañeros, etc. También puede enriquecer sus interacciones en diferentes momentos, circunstancias y con personas de distintos perfiles e idiosincrasias.

De este modo, es posible considerar que las actividades virtuales brindan una alternativa interesante para impulsar el desarrollo de competencias en el ámbito educativo, tanto de índole cognitivo como procedimental y actitudinal, y ofrecen a los docentes de bachillerato recursos novedosos para favorecer la construcción de las competencias establecidas en el perfil de egreso de este nivel educativo.

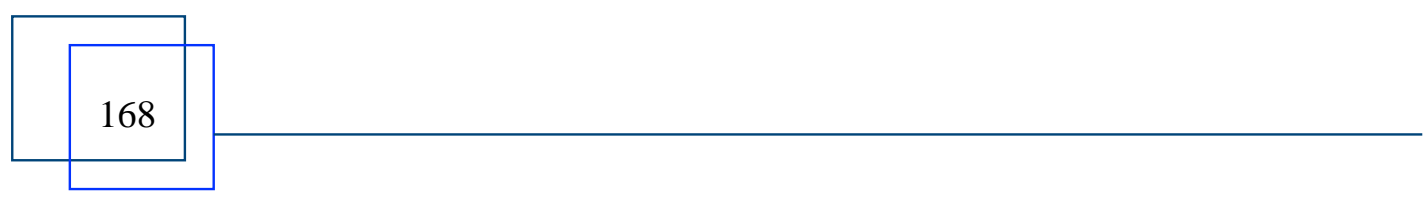




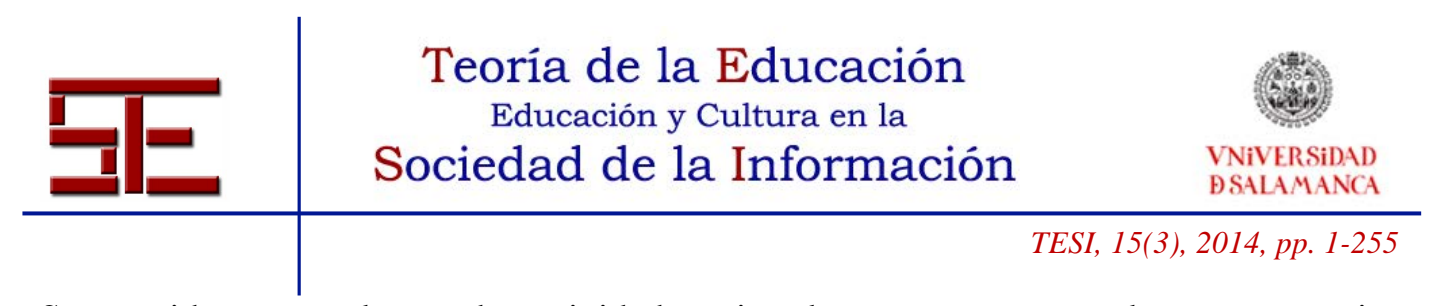

Se considera que el uso de actividades virtuales para promover las competencias sociales puede favorecer el interés y la motivación de los estudiantes debido a que permiten la interactividad, son novedosas y se muestran de manera agradable y atractiva. Así mismo, posibilitan entrar en contacto con compañeros de grupos escolares distintos al que el participante pertenece, lo cual hace factible la ampliación de su círculo de amistades. De igual modo, brindan la posibilidad de expresarse de una manera más abierta y espontánea, sin limitaciones de tiempo.

También ofrecen al docente la oportunidad de interactuar con sus alumnos de manera personalizada, lo cual le permite brindarles ciertas orientaciones sobre su modo de comportarse e interactuar.

\subsection{Sostenibilidad de la propuesta}

Se considera que la implementación de las actividades virtuales para la promoción de las habilidades sociales de estudiantes de preparatoria es sostenible ya que las mismas fueron diseñadas en la plataforma Moodle, la cual es de acceso libre. Esto significa que es suficiente contar con un ordenador con conexión a internet y que tenga instalada esta plataforma para que puedan realizarse las actividades propuestas.

También se aprovecharon los recursos y actividades de Moodle y los programas de Microsoft Office, particularmente Power Point, que es de uso común.

Los diseñadores y tutores del curso no requieren de una capacitación especial para poder implementar las actividades. Es suficiente con que cuenten con conocimientos básicos del uso de la plataforma Moodle y del programa Power Point, así como una formación elemental en tutoría virtual.

Como las actividades son atractivas y estimulantes en sí mismas y no requieren, en general, de la intervención directa de un tutor para su realización, el tiempo necesario para brindar el acompañamiento es reducido. Esto significa que no se requiere una inversión onerosa para el desarrollo, implementación o actualización de estas actividades.

\subsection{Transferibilidad a otros contextos}

Esta propuesta se puede extrapolar a la población de la que se ha obtenido la muestra de estudiantes de la preparatoria en la que se ha realizado el estudio. Ya que las actividades se diseñaron a partir del perfil del adolescente que se encuentra cursando la preparatoria, se considera que las mismas pueden implementarse en instituciones de este nivel educativo, pudiendo generalizarse su aplicación a jóvenes que se encuentren cursando este nivel de estudios. 


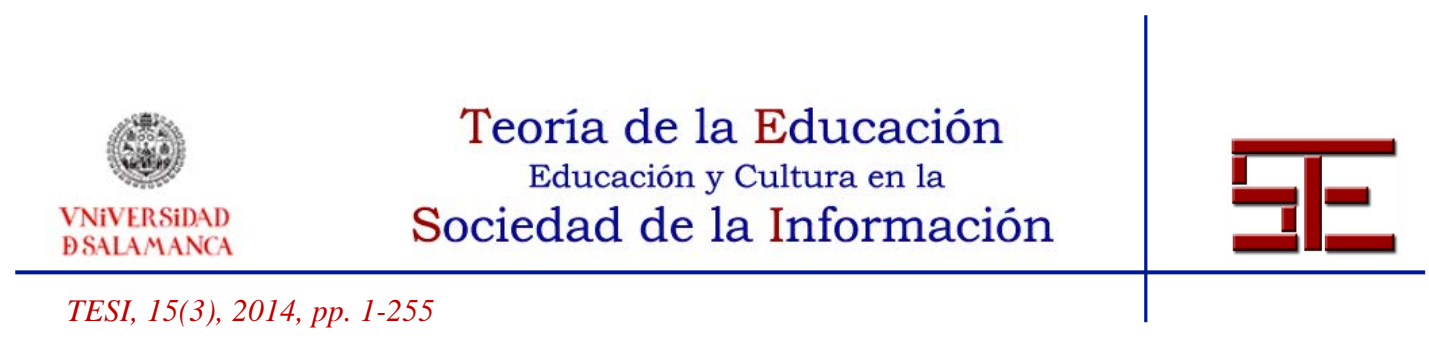

\subsection{Recomendaciones de aplicación}

Resulta conveniente aplicar la escala de habilidades sociales de Goldstein antes y después de la implementación de las actividades virtuales diseñadas en la plataforma Moodle a fin de establecer los perfiles de los participantes en cada uno de los ámbitos evaluados, esto para determinar los niveles de ingreso y egreso de los estudiantes $\mathrm{y}$, de este modo, estar en condiciones de apreciar el impacto que las actividades tienen.

Igualmente, resulta adecuado definir sesiones semanales de trabajo, de preferencia en horario escolar, de este modo se podrá asegurar que los participantes lleven a cabo las actividades y cuenten con las condiciones más propicias para su realización.

También es pertinente contar con tutores que den seguimiento y retroalimentación a las actividades con la intención de asegurar su adecuada realización y de motivar a los estudiantes para que mantengan el interés en las tareas propuestas.

Finalmente, debe disponerse de equipo computacional que soporte la plataforma Moodle y contar con una conexión a internet que asegure el adecuado funcionamiento del curso.

\section{REFERENCIAS}

Aguirre, A. (2004). Capacidad y factores asociados a la resiliencia, en adolescentes del C.E. Mariscal Andres Avelino Caceres del Sectro IV de Pamplona Alta San Juan de Miraflores 2002. Tesis doctoral no publicada. Universidad Nacional Mayor de San Marcos, Lima, Perú.

Alvarado, A. (2003). Diseño instruccional para la producción de cursos en línea y elearning. Docencia Universitaria, 1 (4), 9-24. Recuperado de $<$ http://www.ucv.ve/fileadmin/user_upload/sadpro/Documentos/docencia_vol4_n1_200 3/4_art._1angel_alvarado.pdf $>$.

Bisquerra, R. (2009). Metodología de la investigación educativa. Madrid: La Muralla.

Cacheiro, M. (2011). Recursos educativos TIC de información, colaboración y aprendizaje. Pixel-Bit. Revista de Medios y Educación, 39, 69-81. Recuperado de $<$ http://www.redalyc.org/articulo.oa?id=36818685007>.

Goldstein, A., McGinnis, E., Sprafkin, R., Gershaw, J. y Klein, P. (1997). New strategies and perspectives for teaching prosocial skills. Estados Unidos: McNaughton \& Gunn.

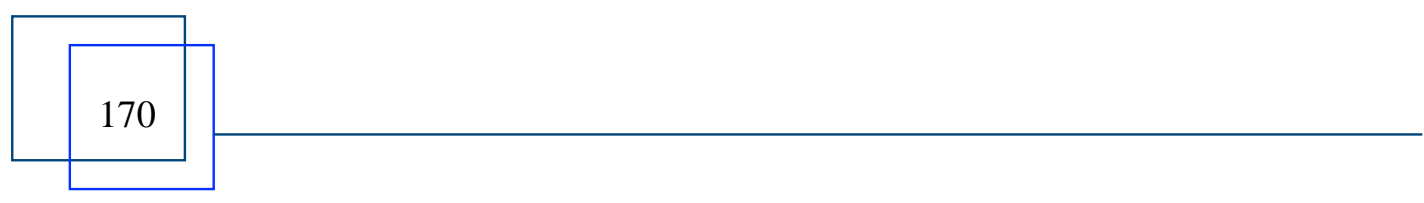




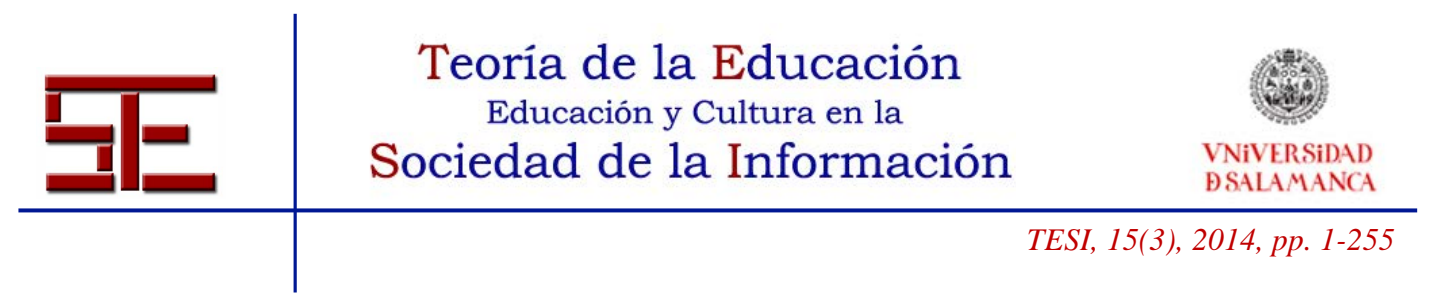

Hernández, R., Fernández-Collado, C. y Baptista, P. (2006). Metodología de la investigación. México: McGrawHill.

Martin, D. (2008). Psicología experimental, Cómo hacer experimentos en psicología. México: CENGAGE Learning.

Monjas, I. y González, B. (1998). Las habilidades sociales en el currículo. España: Ministerio de Educación, Cultura y Deporte. Recuperado de $<$ http://213.0.8.18/portal/Educantabria/RECURSOS/Materiales/Biblinter/HABILIDAD ES.pdf $>$.

Morales, E., Díaz, E. y García, F. (2011). Gestión de objetos de aprendizaje a través de la red, basada en el desarrollo de competencias. Teoría de la Educación. Educación y Cultura en la Sociedad de la Información, 12(1), 99-115. Recuperado de <http://campus.usal.es/ revistas_trabajo/index.php/revistatesi/article/view/7825>.

Navas, M. (coord.). (2009). Métodos, diseños y técnicas de investigación psicológica. Madrid: UNED.

Secretaría de Educación Pública, Estados Unidos Mexicanos (SEP). (2008). Acuerdo número 444 por el que se establecen las competencias que constituyen el marco curricular común. Diario Oficial de la Federación, 21 de octubre de 2008, 1-13. Recuperado de <http://transparencia.info.jalisco.gob.mx/sites/default/files/ACUERDO\%20444.pdf>.

Yukavetsky, G. (2003). La elaboración de un módulo instruccional, Centro de competencias de la comunicación. Puerto Rico: Universidad de Puerto Rico en Humacao. Recuperado de <http://www1.uprh.edu/ccc/CCC/La\%20elaboracion\%20de\%20un\%20modulo\%20instr uccional/CCC_LEDUMI.pdf 